\title{
Welcome Message for Conference
}

Invitation to IBRC 2019

16th INTL BIOPHILIA REHABILITATION CONFERENCE

Okayama, Japan, June 14th,, 2019.

Jose Leon-Carrion

Professor, Ph.D. Chair, Department of Experimental Psychology, University of Seville

IBRA Editorial Board Member and

Conference Chair for IBRC2014 in Seville, Spain.

It is my pleasure to join my esteemed colleagues in welcoming you to the 16th INTERNATIONAL BIOPHILIA REHABILITATION CONFERENCE (IBRC) in Okayama, Japan on June 14th, 2019. This theme for this year's conference is "Preparation of the International Standardization of MOTIVATIVE Exercise Enforcement", chaired by Dr. Yoshiko Morita M.D., Director of the Department of Rehabilitation Medicine at Okayama Rehabilitation Hospital in Okayama City, Japan, and organizing the panel discussion with Professor Takizawa Shigeo (President, International Biophilia Rehabilitation Academy and Professor, Biophilia Institute of the Japanese Ministry of Education, Culture, Sports, Science and Technology) as the panel chair, and Professor Toshiyuki Tanaka (Faculty of Science and Technology, Keio University, Yokohama, Japan) and Director Rika Wada (Tachibana Rehabilitation Service Center, Kobe, Japan) as the panellist.

The IBRC is a leading international forum which has worked for almost 20 years to advance research and diversify techniques for effective rehabilitation programs, with special emphasis on functional recovery in aging populations and reintegration into society. This year's theme for the panel discussion is "The MOTIVATIVE Exercise Program" and its success over conventional passive exercise for improving functional recovery in elderly and disabled persons.

The International Biophilia Rehabilitation Academy (IBRA) is increasingly endorsed each year, with international conferences hosted in Japan, Cuba, Philippines, Spain and Switzerland. Do not miss this exchange of ideas, developments and breakthroughs in rehabilitation strategies.

We look forward to welcoming you in Okayama. 